\title{
Psychometric Evaluation of the Korean version of the Penn State Worry Questionnaire with a Sample of University Students
}

\author{
Lee, Boram \\ Assistant Professor, Department of Early Childhood Education, Woosong University, Daejeon, Korea
}

Purpose: The purpose of this study was to evaluate the psychometric properties and factor structure of the Korean version of the Penn State Worry Questionnaire (PSWQ) for use with the Korean university student population. Methods: A sample of 504 undergraduate students enrolled at a four-year university in South Korea were recruited for the study. Confirmatory factor analysis (CFA) was conducted, based on pre-existing models, to compare the model fit indices of three previously proposed factor structures for the PSWQ. Internal consistency was measured using Cronbach's $\alpha$ while test-retest reliability was assessed using Pearson correlation coefficients. To assess the convergent validity of the PSWQ, Pearson correlations were conducted to examine the association between the PSWQ and the State-Trait Anxiety Inventory-T. Results: CFA results indicated that the one-factor model with method effect fit the data well in the sample. The PSWQ evidenced high internal consistency and adequate to good test-retest reliability over four weeks. The PSWQ also demonstrated substantial convergent validity with trait anxiety. Conclusion: The Korean version of the PSWQ was found to be sufficiently coherent and robust to be used with the university student populations and its reliability and validity has also been supported. The PSWQ measures a facet of worry as a general, unidimensional construct but different response patterns emerged between positively or negatively worded questions.

Key Words: Confirmatory factor analysis; Factor structure; Penn State Worry Questionnaire (PSWQ); Psychometric properties; University students

\section{INTRODUCTION}

\section{Background}

Worry is defined as a conscious, cognitive component of anxiety that involves repetitive thoughts and images about the potentially negative consequences of future events [1]. Worry is also referred to as apprehensive expectations about real-life concerns, such as finances, interpersonal relations, work, school, family health, or chores or other minor matters [2]. Although it is generally an unpleasant experience, worry has been theorized as also serving an adaptive function to the daily challenges of environment [3]. However, excessive levels of worry that are perceived as uncontrollable can become a risk factor or gateway for the development of anxiety disorders [2]. In addition, pathological worry refers to worry that is perceived to be distressing, uncontrollable, and excessive, and it is one of the central features of generalized anxiety disorder (GAD) [4].

One reason why feelings of anxiety and worry are prevalent among university students is that they are in the midst of a major life transition. Certainly, the added pressures of being in a new environment, coping with the stresses and demands of the academic workload, and dealing with the stressful tasks of separation and individuation from their family can also contribute toward causing many students to feel overwhelmed. The experience of worrying associated with anxiety disorders is one of the most common problems facing university students [5]. These risks highlight the need for reliable instruments to screen for associated worry so as to facilitate early detec-

Corresponding author: Lee, Boram https://orcid.org/0000-0003-4796-1565

Department of Early Childhood Education, Woosong University, 171 Dongdaejeon-ro, Dong-gu, Deajeon 34606, Korea. Tel: +82-42-630-9372, Fax: +82-42-630-9369, E-mail: blee@wsu.ac.kr

- This work was supported by Woosong University Research Fund.

Received: Jul 31, 2020 | Revised: Sep 23, 2020 | Accepted: Nov 19, 2020

This is an open access article distributed under the terms of the Creative Commons Attribution Non-Commercial License (http://creativecommons.org/licenses/ by-nc/3.0), which permits unrestricted non-commercial use, distribution, and reproduction in any medium, provided the original work is properly cited. 
tion and timely treatment.

One of the most widely used self-reported measures of worry is the Penn State Worry Questionnaire (PSWQ) [6], which was designed to measure the specific trait of worry in both clinical as well as non-clinical populations. The PSWQ has evidenced excellent psychometric properties such as good internal consistency and construct validity, among older adults [7], community participants [8], multicultural populations $[9,10]$, psychiatric patients [11], and undergraduate students $[12,13]$. Furthermore, the PSWQ has demonstrated adequate test-retest reliability over long periods of time in previous studies (i.e., two to eight weeks) $[6,13,14]$.

Despite the strong psychometric support for the PSWQ, a conflict between the results of various exploratory and confirmatory factor analysis studies has raised questions surrounding the dimensionality of this instrument, leading to an ongoing debate concerning its underlying structure. Meyer et al.[6] found that it is best understood as a unidimensional scale, consistent with its original purpose, but other researchers have identified two correlated orthogonal factors, with positively and negatively worded items form two separate factors $[15,16]$. Yet, other investigators have proposed that a one-factor model that treated the reverse-scored items as a method effect provided better fit than the one- and two-factor models $[8,12,13,17$, $18,19]$. Overall, the many studies of the factor structure of the PSWQ have not yielded a consensus regarding its factor structure. Thus, further investigation is still necessary.

In South Korea (hereafter Korea), a number of studies have performed both exploratory factor analysis (EFA) and confirmatory factor analysis (CFA) on the Korean version of the PSWQ to explore the psychometric properties of this measure. The following results have consistently emerged: (1) the PSWQ has demonstrated high internal consistency $[11,12,19,20]$; (2) the EFA produced a two-factor solution $[19,20]$; (3) comparisons of a single-factor model, a two-factor model, and one-factor model with method effect model using CFA have found that both the two-factor model and the one-factor model with method effect fit the structure of the scale $[12,19]$. However, the total PWSQ exhibited a higher part-whole correlation with positively worded items than with negatively worded ones, and the internal consistency was not adequate for negatively worded items. Therefore, a one-factor model with method effect was deemed the most acceptable option $[12,19]$. Although studies by Park et al. [11], Lim et al. [12], Jeon et al. [19], and Kang et al.[20], have confirmed the psychometric properties of the Korean version of the PSWQ, at least three limitations currently prevent its use in context of Korean uni- versities and research. First, the study by Jeon et al. included both non-psychiatric and psychiatric participants, whereas Park et al. included GAD patients and patients with anxiety disorder; our university student sample may differ in important ways from clinical samples of psychiatric patients. Second, the mean age in the university student sample used by Lim et al was 33.7 years (age range 17 to 45 years), which-although typical of questionnaire validation studies-was a range much broader than that of university students in our study. Moreover, the recruitment strategy used by Lim et al., which targeted student participants from only one discipline (introductory psychology courses), may not guarantee effective representation across a university sample. In contrast to these previous studies, our sample consisted of fairly young university students (mean age 20.6 years) from various disciplines. Third, the study by Kang et al. used the Penn State Worry Questionnaire for children (PSWQ-C), a modified version of the PSWQ, which assesses general characteristics of worry in children and adolescents age 7 to 17[21] and the study's sample consisted of primary school children. Although, the PSWQ-C is administered in a self-report format like the original version, the Likert rating scale is reduced from a 5-point to a 4-point range. Moreover, the PSWQ-C includes 14 of the 16 original items, some of them with modifications in wording so as to make them more developmentally appropriate and readable for children. Due to all these limitations, the previous research studies do not provide sufficient validation of the applicability of the PSWQ with Korean university students. Therefore, a more theoretically sound version of the questionnaire, administered to a more representative Korean university sample, is needed and could provide more reliable data. Furthermore, the use of a universal school-based screening instrument is recommended as an initial step in an effort to provide suitable prevention, diagnosis, and treatment for those currently experiencing from anxiety disorders. In response to these concerns, the aim of the present study was to assess the psychometric properties of and examine the factor structure of the Korean version of the PSWQ by comparing the fits proposed in previously suggested models using CFA in a sample of Korean university students. In addition, the number of participants initially targeted for the current study was approximately 500 .

\section{METHODS}

\section{Participants and Procedures}

This study was conducted during the 2019 2020 aca- 
demic year at a private four-year university in Korea. After the ethical approval, the principal investigator personally contacted the heads of departments and academic instructors from six different disciplines and met with them in person to explain the nature and purpose of the study, and to ask if a survey could be conducted during class time. Once permission was received, the principal investigator made arrangements with academic instructors from six different disciplines for students to complete the questionnaires during scheduled class time. Students from each discipline in all four years of university study were invited to participate. The inclusion criteria required that participating students had to (a) have been born in the Republic of Korea, (b) be currently enrolled at a university, (c) be pursuing an undergraduate degree (d) be willing to participate. Non-Korean citizens and postgraduate students were excluded. The principal investigator visited each class 20 minutes before the end of a class session to explain the purpose of the study and answer any questions from students while they were completing the questionnaire. The participants were given instructions by the principal investigator on how to complete the questionnaire. A paperand-pencil questionnaire and informed-consent forms were distributed to 504 students. On average, the questionnaire took approximately 10 to 15 minutes to complete. The data were collected over a period of four months from October 12 in 2019 to January 12 in 2020.

\section{Ethical Considerations}

The school's Institutional Review Board approved the study procedures (Protocol Code: 1041549-200407-SB-91). The principal investigator had no conflict of interest with the students involved with this research. Participants were assured that their participation in this study was entirely voluntary and could be withdrawn at any point. Written informed consent forms were obtained from all student participants first, and then students completed the questionnaire within the classroom setting at the end of the period. All data including the questionnaires and consent forms were stored securely in a locked filing cupboard in a room that is locked when unoccupied.

\section{Measures}

\section{1) The Penn State Worry Questionnaire}

The PSWQ [6] is a 16-item self-report questionnaire that assesses the intensity and excessiveness of pathological worry. All items are rated on a Likert-type scale ranging from 1 (not at all typical) to 5 (very typical). Eleven items are positively worded (e.g., "When I am under pressure, I worry a lot") and the remaining five items are negatively worded (e.g., "When there is nothing more I can do about a concern, I do not worry about it anymore"). A total score is calculated by summing all items, after reversing the scores for the five negatively worded items. As such, scores range from 16 to 80 , with higher scores indicating greater severity of worry. As mentioned in the introduction, several studies showed excellent internal consistency for the scale in both clinical and non-clinical samples (Cronbach's $\alpha$ s ranging between 0.83 and 0.94) [8,11-16, 22]. The PSWQ also demonstrated adequate test-retest reliability estimates over two to eight weeks $(r=.55-.92)$ in college samples $[6,13,14]$. This study used Lim et al.'s Korean version of the PWSQ, which was verified and validated in Korean university students [12].

\section{2) State-Trait Anxiety Inventory-Trait Form (STAI-T)}

The STAI was designed to assess anxiety as a state at a given point in time (referred as state anxiety) and also to assess trait anxiety, or a stable tendency to experience anxiety, worries, and fears under stressful circumstances frequently at specific moments [22]. In this study, the trait version of the STAI (STAI-T) which consists of 20-items, was used to assess general anxiety proneness. Because pathological worry typically tends to be more associated with trait anxiety [23], the STAI-T was more suitable for and was used in this study. Each item is rated using a 4-point Likert scale format ranging from almost never (1) to almost always (4).

The Korean version of the STAI-T has been validated in Koreans and shown to demonstrate good psychometric properties [24], and Cronbach's $\alpha$ coefficient was 0.81 in the present study.

\section{Statistical Analysis}

The statistical analyses in this study were performed with IBM SPSS Statistics for Windows, Version 23.0 (IBM Corp. Armonk, NY, USA) and AMOS v20. Missing data (less than 5\%) were replaced using the expectation maximization algorithm. Next, CFA with maximum likelihood procedure was used to test a series of alternative plausible models for the structure of the PSWQ. Three competing models suggested in the extant literature were tested, and the resulting fit indices were compared to determine which one provided the best fit to our data. Model 1 was the original unidimensional, one-factor model obtained by Meyer et al. [6], in which all 16 items loaded onto a single factor. Model 2 was the two-factor model of Fresco 
et al. [15], with the 11 positively worded items loaded on the first factor and the 5 negatively worded items loaded on the second factor, and in which both factors were allowed to correlate. The final model tested was a one-factor model that included a negative wording method effect, with all 16 items as indicators of a latent worry factor and the 5 negatively worded items as indicators of a latent method factor, hypothesized by Hazlett-Stevens et al. [18]

The model fit was evaluated by the relative chi-square $\left(x^{2}\right)$, comparative fit index (CFI), goodness-of-fit index (GFI), root mean square error of approximation (RMSEA), and standardized root mean square residual (SRMR). The relative $x^{2}$ is the ratio of $x^{2}$ to the number of degrees of freedom $\left(x^{2} / \mathrm{df}\right)$, and its recommended reference value for an acceptable model fit is less than 5 [25]. For the CFI and GFI, values equal to or greater than 0.90 are considered indicative of adequate fit [26]. In practice, RMSEA values of less than 0.05 indicate an excellent fit, although values between 0.05 and 0.08 reflect reasonable errors of approximation [27]. SRMR values below 0.05 suggest good model fit $[25,26]$. In addition, the $x 2$ difference test was applied to compare the differences between the alternative models in CFA. Although, there is no clear consensus regarding the rules of thumb recommended in the sample size, one suggested guideline is to have 5 or 10 participants per estimated parameter [28]. On the other hand, others have indicated that 300 is a good sample size for a CFA [29]. Therefore, the sample size of 504 satisfies the estimated and minimum sample size requirement for CFA.

Cronbach's alpha coefficient $(\alpha)$ was calculated to estimate the internal consistency. Next, test-retest reliability of the PSWQ was examined using Pearson's correlation between scores on two separate administrations of the PSWQ, four weeks apart, with a subsample of 30 student participants. Finally, to assess the convergent validity of the PSWQ, the associations between the PSWQ and the criteria instrument STAI-T were examined using Pearson's r.

\section{RESULTS}

\section{Descriptive Statistics}

A total of 504 undergraduate students (167 males and 337 females) completed the Korean version of the PSWQ. The participants' age ranged from 18 to 28 years ( $M=20.2$, $\mathrm{SD}=1.63)$. The great majority $(85.5 \%)$ were in the 19 22 age range. The mean age for male students were 20.6 years $(S D=2.06)$ and 20.0 years for female students $(S D=1.34)$. Students were enrolled from a variety of arts and sciences disciplines, with social work the most common $(n=135$, $26.8 \%)$, followed by design ( $\mathrm{n}=100,19.8 \%)$, culinary arts $(\mathrm{n}=99,19.6 \%)$, education $(\mathrm{n}=99,19.6 \%)$, public health $(\mathrm{n}=50$, $9.9 \%)$, and architecture $(\mathrm{n}=21,4.2 \%)$. The overall mean of the PSWQ scores was 47.90 (SD=12.38; range 17 80) (Table 1).

\section{Confirmatory Factor Analysis}

As can be seen in Table 1, the one-factor model of the PSWQ showed a rather poor fit to the data, with values for CFI, RMSEA, and SRMR that were outside the recommended cutoffs $\left(x^{2}=663.0, \mathrm{dF}=104 ; x^{2} / \mathrm{dF}=6.4 ; \mathrm{CFI}=0.86 ; \mathrm{GFI}=0.88\right.$; RMSEA $=0.103(90 \% \mathrm{CI}=0.096 \sim 0.111)$; SRMR=0.087). By contrast, the two-factor model (consisting of worry and absence of worry) was found to provide a good fit to the data $\left(x^{2}=354.7, \mathrm{dF}=103 ; x^{2} / \mathrm{dF}=3.4 ; \mathrm{CFI}=0.93 ; \mathrm{GFI}=0.94\right.$; RMSEA $=0.070(90 \% \mathrm{CI}=0.062 \sim 0.078)$; SRMR=0.052), although the value for SRMR was marginally above the recommended criterion of 0.50 to indicate good fit. Finally, the one-factor model with method factor solution was found to fit the data slightly better than the two-factor model $\left(x^{2}=334.7, \mathrm{df}=\right.$ 99; $x^{2} / \mathrm{df}=3.4 ; \mathrm{CFI}=0.94 ; \mathrm{GFI}=0.94 ; \mathrm{RMSEA}=0.069(90 \%$ $\mathrm{CI}=0.061 \sim 0.077)$; $\mathrm{SRMR}=0.036$ ), as evidenced by a decrease in the $x 2$ value and the improved CFI, RMSEA and SRMR statistics, which were in the good fit range. Although the last two models were generally acceptable, only the final model had an SRMR of less than 0.05 . An additional $x^{2}$ difference test indicated that the one-factor model with method effect provided a better fit than either the one-factor model $\left(\Delta x^{2}(5)=328.3, p<.001\right)$ or the two-factor model $\left(\Delta x^{2}(4)=20.0, p<.001\right)$. In general, the best results on all goodness-of-fit indices were obtained with the one-factor model with method effect (Figure 1). The suitability of this model can furthermore be determined by its parameter

Table 1. Goodness-of-fit Indices for PSWQ Models

\begin{tabular}{lcccccccc}
\hline Models $^{1}$ & $\mathrm{k}$ & $x^{2}$ & $\mathrm{df}$ & $x^{2} / \mathrm{df}$ & CFI & GFI & RMSEA (90\% CI) & SRMR \\
\hline Model 1 & 16 & $663.0^{*}$ & 104 & 6.4 & 0.86 & 0.88 & $0.103(0.096 \sim 0.111)$ & 0.087 \\
Model 2 & 16 & $354.7^{*}$ & 103 & 3.4 & 0.93 & 0.94 & $0.070(0.062 \sim 0.078)$ & 0.052 \\
Model 3 & 16 & $334.7^{*}$ & 99 & 3.4 & 0.94 & 0.94 & $0.069(0.061 \sim 0.077)$ & 0.036 \\
\hline
\end{tabular}

$\mathrm{k}=$ number of items; $\mathrm{df}=$ degrees of freedom; $\mathrm{CFI}=$ comparative fit index; $\mathrm{GFI}=$ goodness of fit index; RMSEA=root mean square error of approximation; SRMR=standardized root mean residual; Model 1=One-factor model; Model 2=Two-factor model; Model 3=One-factor model with method effect; ${ }^{*} p<.01$. 


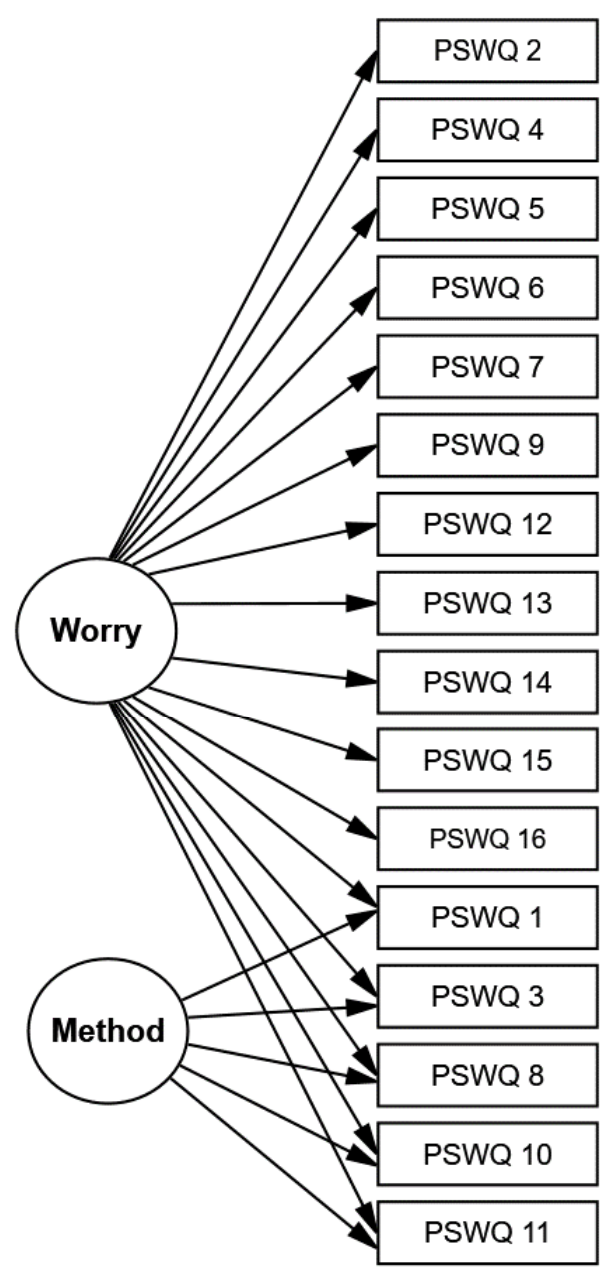

Figure 1. One factor model with method effect of the PSWQ.

estimates. All items loaded positively on each factor and statistically significant, with values from 0.31 to 0.88 (Table 2).

\section{Reliability}

Internal consistency tests provided a Cronbach's $\alpha$ of 0.89 for the entire scale, with an $\alpha$ of 0.94 for the 11 positively worded items and 0.68 for the 5 negatively worded items. The test-retest correlations for the total PSWQ the 11 positively stated items, and the 5 negatively items, respectively, were $\mathrm{r}=0.81(p<.01), \mathrm{r}=0.78(p<.01)$, and $\mathrm{r}=0.60(p<.01)$, with a four-week interval between test administrations.

\section{Convergent Validity}

As expected, the PSWQ and STAI-T showed a positive and moderate correlation $(\mathrm{r}=0.58, p<.01)$, thus providing evidence for convergent validity. The overall PSWQ score
Table 2. Standardized Factor Loadings for the Tone-factor, Two-factor, and One-factor with Method Effect Models of the PSWQ

\begin{tabular}{|c|c|c|c|c|c|}
\hline \multirow{2}{*}{$\begin{array}{l}\text { PSWQ } \\
\text { items }\end{array}$} & \multirow{2}{*}{$\begin{array}{l}\text { Model } 1 \\
\text { Factor } 1 \\
\end{array}$} & \multicolumn{2}{|c|}{ Model 2} & \multicolumn{2}{|c|}{ Model 3} \\
\hline & & Factor 1 & Factor 2 & Factor 1 & Factor 2 \\
\hline 2 & 0.39 & 0.39 & & 0.39 & \\
\hline 4 & 0.56 & 0.56 & & 0.56 & \\
\hline 5 & 0.39 & 0.39 & & 0.39 & \\
\hline 6 & 0.69 & 0.69 & & 0.69 & \\
\hline 7 & 0.42 & 0.42 & & 0.42 & \\
\hline 9 & 0.47 & 0.46 & & 0.46 & \\
\hline 12 & 0.76 & 0.76 & & 0.75 & \\
\hline 13 & 0.59 & 0.59 & & 0.60 & \\
\hline 14 & 0.88 & 0.89 & & 0.85 & \\
\hline 15 & 0.54 & 0.54 & & 0.55 & \\
\hline 16 & 0.70 & 0.70 & & 0.69 & \\
\hline 1 & 0.54 & & 0.45 & 0.54 & 0.33 \\
\hline 3 & 0.40 & & 0.44 & 0.40 & 0.41 \\
\hline 8 & 0.44 & & 0.32 & 0.44 & 0.38 \\
\hline 10 & 0.45 & & 0.31 & 0.45 & 0.52 \\
\hline 11 & 0.50 & & 0.47 & 0.50 & 0.42 \\
\hline
\end{tabular}

Model 1 is a one-factor model with all items loading on one factor. Model 2 is two-factor model in which positively and negatively worded items load on two separate factors. Model 3 is a one-factor model with method effect with all 16 items load on one factor and the five negatively worded items load on a method factor.

had an extremely high correlation with the positively worded items $(\mathrm{r}=0.94, p<.01)$. The total STAI-T score had a moderate correlation with the positively worded items $(\mathrm{r}=0.48, p<.01)$. While the correlation between the total PSWQ score and the negatively worded items was moderate $(\mathrm{r}=0.45, p<.01)$, the correlation between the negatively worded items and the total STAI-T score was relatively low $(\mathrm{r}=0.26, p<.01)$. Lastly, positively and negatively stated items of the PSWQ exhibited a very weak correlation with each other $(\mathrm{r}=0.16, p<.01)$. These results suggest that the positively worded items of the PSWQ had a stronger positive correlation with the total PSWQ and STAI-T than the negatively worded items of the PSWQ.

\section{DISCUSSION}

The purpose of the present study was to evaluate the factor structure and psychometric properties of the Korean version of the PSWQ when administered to Korean uni- 
versity students. The results indicate that the PSWQ is a reliable and valid instrument for assessing symptoms of anxiety in Korean university students. First, the overall mean score was higher than the means obtained by Lim et al. [12] with Korean university students $(M=45.35, S D=$ 11.73) and Carter et al. [9] with African-American college students $(M=42.45, S D=10.64)$. However, it was lower than the means for samples of American college students $(M=48.39, S D=13.87)$ as found by Fresco et al. [15]. Next, the PSWQ's reliability was adequate; the results are in accordance with those obtained using translations of the scale into other languages $[13,19]$. The PSWQ was demonstrated to possess adequate test-retest reliability with regard to both the total score and the two separate factors over a period of four weeks.

With regards to factor structure, the CFA supported the two-factor model and the one-factor model with method effect. However, the one-factor model with method effect related to negatively stated items yielded better fit indices overall. Our results are in agreement with the results of some previous studies, which revealed that the one-factor model with method effect provided the best overall model fit to the data compared to the two-factor model $[8,12,13$, 17-19]. Also, this model is considered to be preferable from a theoretical point.

In support of this, the positively worded items had a significant relationship with the total PSWQ, suggesting that this positively worded item factor is closely associated with a similar underlying structure of the PSWQ. Moreover, the moderate correlation between this positively worded factor and the STAI-T indicates that this factor is measuring a trait similar to the theoretical construct of general anxiety or vice versa. However, the negatively worded items had only a moderate correlation with the total PSWQ scores and a weak correlation with the STAI-T, suggesting that this factor, which consists of reverse-scored items, should be considered a statistical artifact and is not really meaningful. Hence, it is reasonable to conclude that the PSWQ has a single construct of worry and that the second factor results from the effect of the reverse wording and does not indicate the existence of another meaningful worry construct [17-19]. This conclusion is reinforced by the fact that the internal consistency of the 11 positively worded items was higher than that of the total PSWQ scale, whereas the internal consistency of the 5 negatively stated items was noticeably lower.

These findings raise the question of whether the reverse wording of the negative items is helpful, as their inclusion complicates the interpretation of the PSWQ. It has been suggested that the easiest way to deal with the methodo- logical artifact of reverse-scored items is to design scales without such items and instead employ an abbreviated scale that includes only positively worded items $[17,18]$. Such a procedure would improve the validity of the PSWQ's unidimensional structure [10]. However, the removal of these items may be especially problematic with participants who exhibit an affirmative response bias. Rather, the presence of reverse-scored items may be useful in determining whether such a bias is present [18].

The current study results provide an important implication for assessing anxiety in students. The availability of a valid instrument such as the PSWQ is critical for nurses and other health professionals who work with university students to recognize those who are at a greater risk of experiencing anxiety disorder. Use of this tool can promote nursing interventions that can be appropriately planned, implemented, and evaluated.

There are a few limitations to this study worth mentioning. First, the sample consisted of only undergraduate students, so we must be cautious when generalizing the results to other age groups or populations. Prior studies suggest that the factor structure of the PSWQ may differ across various cultural groups, clinical populations, or age groups $[6,18]$. Thus, the findings should be tested with different and more heterogeneous populations to provide more support for the factor structure of the PSWQ. Second, the current data were obtained through self-report measures, which may be susceptible to shared method variance and response bias. Future studies using multimethod assessments would address this issue. Finally, no attempt was made to recruit participants with a history of psychiatric or anxiety disorders. This is an important issue because worry is naturally present in all anxiety disorders and a central feature of GAD. Indeed, the PSWQ can be used as a screening tool to detect anxiety disorder [30]. Therefore, future studies could examine the utility of the PSWQ across diverse non-college-aged and nonclinical populations as well as clinical samples.

\section{CONCLUSION}

Notwithstanding these limitations, our results largely confirm the conclusions reached by previous psychometric studies of the PSWQ. The PSWQ appears to be a useful tool for measuring worry and it demonstrated adequate reliability and validity with a sample of Korean university students. As in other studies with university student samples, a one-factor model that accounted for the effect of negatively worded items provided the best fit to the data. However, participants' responses to items on the PSWQ 
may vary according to whether they are reporting the degree to which worry is present or absent $[18,19]$. It may thus be beneficial to test for a method factor when considering the reliability of other measures that contain a similar combination of positively and negatively worded items.

\section{CONFLICTS OF INTEREST}

The author declared no conflicts of interest.

\section{ORCID}

Lee, Boram

https://orcid.org/0000-0003-4796-1565

\section{REFERENCES}

1. Vasey MW, Daleiden EL. Worry in children. In: Davey GCL, Tallis F, editors. Worrying: perspectives on theory, assessment and treatment. Chichester: Wiley; 1994. 207 p.

2. Barlow DH. Anxiety and its disorders: the nature and treatment of anxiety and panic. 2nd ed. New York: Guilford Press; 2002. 704 p.

3. Borkovec TD. The nature, functions, and origins of worry. In: Davey GCL, Tallis F, editors. Worrying: perspectives on theory, assessment and treatment. Chichester: Wiley; 1994. 33 p.

4. American Psychiatric Association. Diagnostic and statistical manual of mental disorders. 5th ed Arlington, VA: American Psychiatric Publishing Inc; 2013. 991 p.

5. Pedrelli P, Nyer M, Yeung A, Zulauf C, Wilens T. College students: Mental health problems and treatment considerations. Academic Psychiatry. 2015;39(5):503-511. https://doi.org/10.1007/s40596-014-0205-9

6. Meyer TJ, Miller ML, Metzger RL, Borkovec TD. Development and validation of the Penn State Worry Questionnaire. Behaviour Research and Therapy. 1990;28(6):487-495. https://doi.org/10.1016/0005-7967(90)90135-6

7. Wuthrich VM, Johnco C, Knight A. Comparison of the Penn State Worry Questionnaire (PSWQ) and abbreviated version (PSWQA) in a clinical and non-clinical population of older adults. Journal of Anxiety Disorders. 2014;28(7):657-663. https://doi.org/10.1016/j.janxdis.2014.07.005

8. Jiménez-Ros AM, Carmona-Márquez J, Pascual LM. Pathological worry in Portugal: the Portuguese version of the Penn State Worry Questionnaire (PSWQ). The Spanish Journal of Psychology. 2019;22(e63):1-8.

https://doi.org/10.1017/sjp.2019.61

9. Carter MM, Sbrocco T, Miller Jr O, Suchday S, Lewis EL, Freedman REK. Factor structure, reliability, and validity of the Penn State Worry Questionnaire: differences between AfricanAmerican and White-American college students. Journal of Anxiety Disorders. 2005;19(8):827-843.

https://doi.org/10.1016/j.janxdis.2004.11.001
10. Castillo C, Macrini L, Cheniaux E, Landeira-Fernandez J. Psychometric properties and latent structure of the Portuguese version of the Penn State Worry Questionnaire. The Spanish Journal of Psychology. 2010;13(1):431-443.

https://doi.org/10.1017/s113874160000398x

11. Park HJ, Kim JH, Lee JH, Heo JY, Yu BH. The usefulness of the Korean version of Penn State Worry Questionnaire for screening generalized anxiety disorder: a receiver operating characteristic analysis. Psychiatry Investigation. 2014;11(1):12-17. https://doi.org/10.4306/pi.2014.11.1.12

12. Lim YJ, Kim YH, Lee EH, Kwon SM. The Penn State Worry Questionnaire: psychometric properties of the Korean version. Depression and Anxiety. 2008;25(10):E97-E103.

https://doi.org/10.1002/da.20356

13. Zhong J, Wang C, Li J, Liu J. Penn State Worry Questionnaire: structure and psychometric properties of the Chinese version. Journal of Zhejiang University SCIENCE B. 2009;10(3):211-218. https://doi.org/10.1631/jzus.B0820189

14. Yilmaz AE, Gençöz T, Wells A. Psychometric characteristics of the Penn State Worry Questionnaire and Metacognitions Questionnaire-30 and metacognitive predictors of worry and obsessive-compulsive symptoms in a Turkish sample. Clinical Psychology and Psychotherapy. 2008;15(6):424-439. https://doi.org/10.1002/cpp.589

15. Fresco DM, Heimberg RG, Mennin DS, Turk CL. Confirmatory factor analysis of the Penn State Worry Questionnaire. Behaviour Research and Therapy. 2002;40(3):313-323. https://doi.org/10.1016/S0005-7967(00)00113-3

16. Wu SM, Schuler TA, Edwards MC, Yang HC, Brothers BM. Factor analytic and item response theory evaluation of the Penn State Worry Questionnaire in women with cancer. Quality of Life Research. 2013; 22(6):1441-1449. https://doi.org/10.1007/s11136-012-0253-0

17. Brown TA. Confirmatory factor analysis of the Penn State Worry Questionnaire: multiple factors or method effects? Behaviour and Research Therapy. 2003;41(12):1411-1426. https://doi.org/10.1016/S0005-7967(03)00059-7

18. Hazlett-Stevens H, Ullman JB, Craske MG. Factor structure of the Penn State Worry Questionnaire: examination of a method factor. Assessment. 2004;11(4):361-370. https://doi.org/10.1177/1073191104269872

19. Jeon JW, Kim D, Kim E, Roh S. Exploratory and confirmatory factor analysis of the Korean version of the Penn State Worry Questionnaire. Anxiety and Mood. 2017;13(2):86-92. https://doi.org/10.24986/anxmod.2017.13.2.86

20. Kang SG, Shin JH, Song SW. Reliability and validity of the Korean version of the Penn State Worry Questionnaire in primary school children. Journal of Korean Medical Science. 2010; 25(8):1210-1216. https://doi.org/10.3346/jkms.2010.25.8.1210 21. Chorpita BF, Tracey SA, Brown TA, Collica TJ, Barlow DH. 
Assessment of worry in children and adolescents: an adaptation of the Penn State Worry Questionnaire. Behaviour Research and Therapy. 1997;35(6):569-581.

https://doi.org/10.1016/s0005-7967(96)00116-7

22. Spielberger CD, Gorsuch RL, Lushene R, Vagg PR, Jacobs GA. Manual for the State-Trait Anxiety Inventory. Palo Alto, CA: Consulting Psychology Press; 1983. 3 p.

23. Kelly WE. Anxiety and stress as contributory factors in pathological and nonpathological worry. Psychology Journal. 2008; 5(3):147-157.

24. Lim YJ, Lee SY, Kim JH. Distinct and overlapping features of anxiety sensitivity and trait anxiety: the relationship to negative affect, positive affect, and physiological hyperarousal. Korean Journal of Clinical Psychology. 2005;2:439-449.

25. Marsh HW, Hau KT, Wen Z. In search of golden rules: comment on hypothesis testing approaches to setting cutoff values for fit indexes and dangers in overgeneralizing Hu \& Bentler's
(1999) findings. Structural Equation Modeling. 2004;11(3):320-341. https://doi.org/10.1207/s15328007sem1103_2

26. Fan X, Sivo SA. Sensitivity of fit indices to model misspecification and model types. Multivariate Behavior Research. 2007; 42(3):509-529. https://doi.org/10.1080/00273170701382864

27. Browne MW, Cudeck R. Alternative ways of assessing model fit. Sociological Methods and Research. 1992;21(2):230-258. https://doi.org/10.1177/0049124192021002005

28. Bollen KA. Structural equations with latent variables. New York: John Wiley and Sons Inc; 1989. 528 p.

29. Comrey AL, Lee HB. A first course in factor analysis 2 nd ed. Hillsdale, NJ: Lawrence Erlbaum; 1992. 442 p.

30. Behar E, Alcaine O, Zuellig AR, Borkovec TD. Screening for generalized anxiety disorder using the Penn State Worry Questionnaire: a receiver operating characteristic analysis. Journal of Behavior Therapy and Experimental Psychiatry. 2003;34(1): 25-43. https://doi.org/10.1016/s0005-7916(03)00004-1 